\title{
Characterization of solid hydrogen targets
}

M.C. Fujwwara ${ }^{1, *}$, J.M. Bailey ${ }^{2}$, G.A. Beer ${ }^{3}$, J.L. Beveridge ${ }^{4}$, J.L. Douglas ${ }^{3}$,

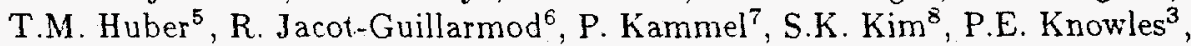
A.R. Kunselman ${ }^{9}$, M. Maier ${ }^{3}$, G.M. Marshall ${ }^{4}$, C.J. Martof ${ }^{10}$, G.R. Mason ${ }^{3}$, F. Mulhauser ${ }^{4, t}$, A. Olin $^{3}$, C. Petitjean ${ }^{11}$, T.A. Porcelli ${ }^{3}$ and J. Zmeskal ${ }^{12}$

${ }^{1}$ University of British Columbia, Vancouver, British Columbia. V6T 2A6 Canada,

${ }^{2}$ Chester Technology, England,

${ }^{3}$ University of Victoria, Victoria, British Columbia, V8W 2 Y2 Canada,

${ }^{4}$ TRIUMF, Vancouver, British Columbia, V6T $2 A 3$ Canada,

${ }^{5}$ Gustavus Adolphus College, St. Peter, MN 56082, USA,

${ }^{6}$ Université de Fribourg, $\mathrm{CH}-1700$ Fribourg, Switzerland,

${ }^{7}$ Laurence Berkeley Laboratory, Berkeley: CA 94720, USA,

${ }^{8}$ Jeonbuk National University, Jeonju City 560-756, S. Korea,

${ }^{9}$ University of Wyoming, Laramie, WY 82071, USA,

${ }^{10}$ Temple University, Philadelphia, PA 19122, USA,

${ }^{11}$ Paul Scherrer Institute, CH-5232 Villigen, Switzerland,

${ }^{12}$ Austrian Academy of Sciences, A-1090 Wien, Austria

In experiments using the TRIUMF solid hydrogen target system, the knowledge of the target thickness and uniformity is often essential in order to extract physical parameters from the data. We have characterized the thickness and uniformity of frozen targets using the energy loss of alpha particles. An accuracy of $\sim 5 \%$ was achieved, a limit imposed by the uncertainty in the stopping powers. The details of the method are described, and the thickness calibration of the target is presented.

Keywords: muon catalyzed fusion, thickness measurement, solid hydrogen.

*e-mail: fujiwara@triumf.ca

'Present address: Université de Fribourg, CH-1700 Fribourg, Switzerland 


\section{Introduction}

A target system for solid hydrogen developed at TRIUMF $[1,2]$ has proven to be a powerful tool in muon catalyzed fusion research. Several measurements have been made [3] and more are proposed for the future $[4,5,6]$. One of the advantages of the thin film targets is that one can perform cross section measurements using a "beam" of $\mu d, \mu t[3,4]$, or possibly $\mu a[5,6]$. In such measurements, knowledge of film thickness and uniformity is essential, since the uncertainty in the thickness directly enters in the final result.

The thicknesses of our hydrogen targets range from a few $\mu \mathrm{g} \cdot \mathrm{cm}^{-2}(\sim 1 \mu \mathrm{m})$ to a few $\mathrm{mg} \cdot \mathrm{cm}^{-2}$. Conventional thin film methods, such as optical interferometry, would not work due to the cryogenic and spatial constraints. The energy loss of alpha particles passing through the layer was used to measure the target thickness and uniformity.

\section{Measurement}

The energy of alpha particles traversing a solid hydrogen film was measured with a charged particle detector. With the knowledge of the stopping power, the energy lost in the film can be converted to a thickness.

Fig. 1 shows a schematic view of the experimental setup. The general description of the TRIUMF target system is given in Refs. [1,2]. Americium-241 was electrodeposited onto a gold-plated oxygen-free copper plate to form a custommanufactured ${ }^{1}$ vertical array of five spot sources, each separated by $10 \mathrm{~mm}$ center to center. The target plate was cooled to approximately $3 \mathrm{~K}$, and the hydrogen gas solidified upon it when introduced through the gas diffuser which could move up and down. Although different deposition systems have been developed, the present measurements used a perforated foil gas diffuser [1] which was placed at a distance of about $14 \mathrm{~mm}$ from the cold plate. The results from other systems will be reported elsewhere [7]. Alpha particles penetrating the hydrogen film were detected by a silicon detector, which was mounted on the frame of the retractable diffuser. The detector can thus move vertically to measure the alpha energy spectrum at the five different positions. The detector was collimated in order to reduce the angular dispersion of the alpha particles and to accept alphas from only one spot source at a time. A energy resolution of $\sim 0.4 \%$ at a detector temperature around $90 \mathrm{~K}$ was achieved.

The amount of gas injected into the system is quoted in units of Torr-litres (TL), where one Torr-litre corresponds to the number of molecules in one litre of gas at a pressure of one Torr and ambient temperature $(295 \mathrm{~K})$.

\footnotetext{
${ }^{1}$ Isotope Products Laboratories, 1800 N. Keystone St. Burbank, CA, USA.
} 


\section{DISCLAIMER}

This report was prepared as an account of work sponsored by an agency of the United States Government. Neither the United States Government nor any agency thereof, nor any of their employees, makes any warranty, express or implied, or assumes any legal liability or responsibility for the accuracy, completeness, or usefulness of any information, apparatus, product, or process disclosed, or represents that its use would not infringe privately owned rights. Reference herein to any specific commercial product, process, or service by trade name, trademark, manufacturer, or otherwise does not necessarily constitute or imply its endorsement, recommendation, or favoring by the United States Government or any agency thereof. The views and opinions of authors expressed herein do not necessarily state or reflect those of the United States Government or any agency thereof. 


\section{DISCLAIMER}

Portions of this document may be illegible in electronic image products. Images are produced from the best available original document. 


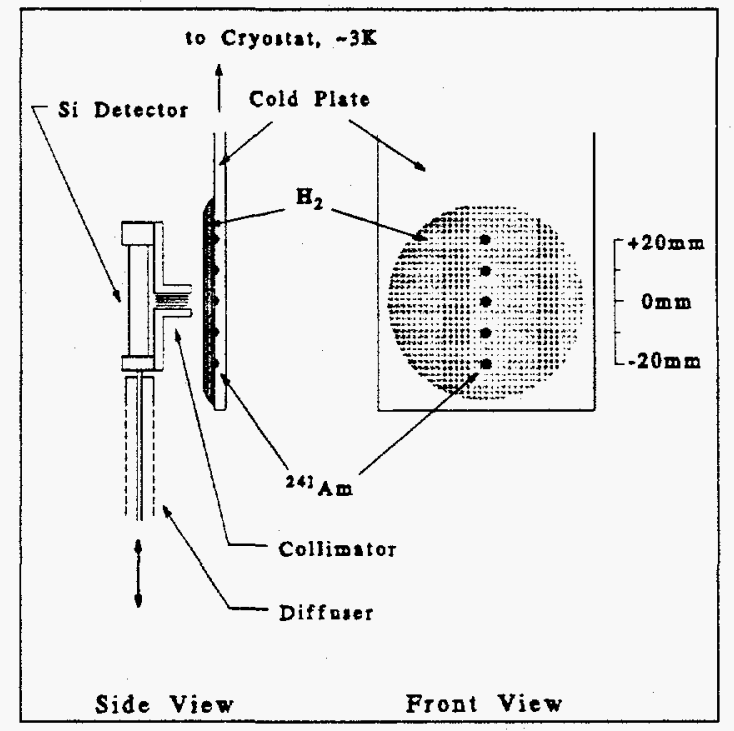

Figure 1: Schematic views of the experimental setup.

The mean energy value $\langle E\rangle$ was determined from the distribution function $f(E)$ in the alpha particle energy spectrum via:

$$
<E>=\frac{\int_{\langle E\rangle-\epsilon}^{\langle E\rangle+\epsilon} f(E) E d E}{\int_{\langle E\rangle-\epsilon}^{\langle E\rangle+\varepsilon} f(E) d E},
$$

where $\epsilon$ is a finite cutoff value. If we know $\operatorname{Range}(E)$, the range of alpha particles as a function of energy, the thickness of the target can be obtained by:

$$
\text { Thickness }=\operatorname{Range}\left(\left\langle E_{\text {init }}\right\rangle\right)-\operatorname{Range}\left(\left\langle E_{\text {fin }}\right\rangle\right) \text {, }
$$

where $\left\langle E_{\text {init }}>\right.$ is the initial energy of the alpha particles and $\left\langle E_{f \text { in }}>\right.$, the energy after traversing the target.

Accurate knowledge of stopping power is important in order to determine the thickness by energy loss. Detailed discussions of the slowing down process of charged particles and a comparison of different stopping power and range tables are found in Ref. [5]. Two recent compilations by Ziegler et al. [8] and by ICRU [9] are in good agreement with each other for the energies required for the measurement. The ICRU table was employed for the analysis.

Estimating the uncertainties in the stopping powers is difficult, since no experimental data are available for heavy charged particles slowing in the solid state 


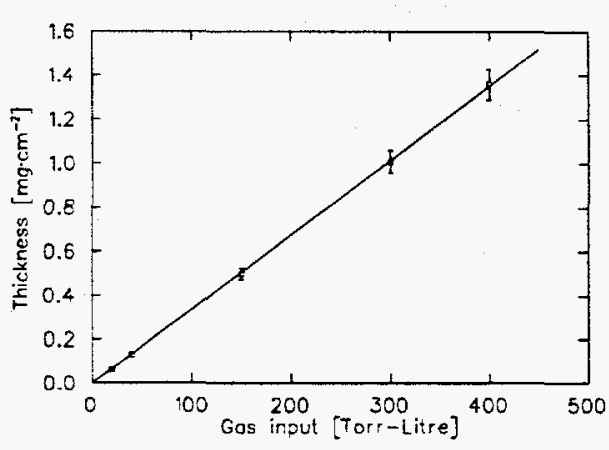

Figure 2: Linearity of deposition. Thickness is plotted as a function of the injected gas. The solid line is a least-square fit to the data points.

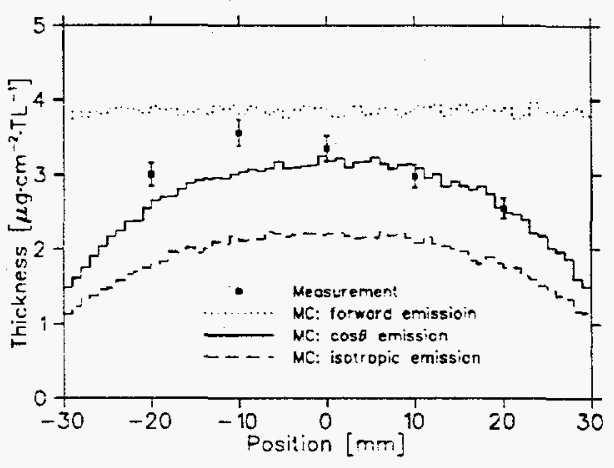

Figure 3: The measured thickness profile for hydrogen targets, compared with Monte Carlo calculations with different emission models.

of hydrogen (except at a few keV energies). After a detailed survey of the physical phase effect on the stopping power [5], a conservative uncertainty of $5 \%$ is quoted. This uncertainty limits the precision of the measurement.

Other sources of systematic uncertainties were investigated, but found to be small compared to the above. These include the effect of cut-off value $\epsilon$ in Eq. 1, the angular dispersion of the alpha path, and calibration of the detector. The systematic errors from these sources, as well as the statistical error, were added quadratically to obtain the total uncertainty.

\section{Results}

Measurements were made with films made from different input gas and with different deposition conditions. Data for the central source spot from various protium films are plotted in Fig. 2. The solid line is a least-square fit to the data, which confirms the linearity of the deposition. Similarly, the other spots on the target show a very good linearity. There is no evidence of saturation in the thickness for increasing gas input up to 400 Torr-litres, permitting the extrapolation to thicker targets which cannot be directly measured due to the limited range of the 5.5 $\mathrm{MeV}$ alpha particles.

The calibration factor, target thickness per unit gas inputs, was determined from the slope of the linear fit, and the values for each spot are presented in Table 1 for both protium and neon films. The calibration factors for deuterium and tritium must be multiplied by 2 and 3 respectively, due to the molecular weight difference. 


\section{Discussion}

The results shown in Table 1 should be used with caution, when applied to beam experiments. Due to non-uniformity; the average thickness of a target depends on the width and profile of the beam which stops in the target. An effective average thickness is defined via:

$$
T^{e f f}=\frac{\sum w_{i} T_{i}}{\sum w_{i}}
$$

where $T_{i}$ is the thickness at the $i$ th source spot, and $w_{i}$ the weighting factor. A weighted root-mean-square deviation of thickness is defined via:

$$
\Delta T_{r m s}^{\varepsilon f f}=\sqrt{\frac{\sum w_{i}\left(T_{i}-T^{\epsilon f f}\right)^{2}}{\sum w_{i}}} .
$$

Assuming a gaussian beam distribution with a $30 \mathrm{~mm}$ FWHM, for example, and in an approximation considering only the vertical dimension, we obtain $T^{e f f}=$ $3.20 \mu \mathrm{g} \cdot \mathrm{cm}^{-2}$ per Torr-lite gas input with $\Delta T_{r m s}^{\epsilon f f}=0.31 \mu \mathrm{g} \cdot \mathrm{cm}^{-2}$ for a protium target.

In order to better understand the mechanism of film deposition, Monte Carlo simulations were performed with the following assumptions: molecules originate uniformly from the gas diffuser surface, they stick to the cold surface at the position of first contact, and there is no interaction between the molecules. The last assumption can be justified from the requirement to maintain low pressure during deposition to keep the target film temperature cold, i.e., a significant intermolecular interaction would cause heat conduction from the diffuser $(\sim 90 \mathrm{~K}$ ) to the target foil $(\sim 3 \mathrm{~K})$. Three different models for the angular distribution of molecules emitted from the diffuser surface were used in simulations; (1) the forward emission model assuming $\theta=0$, where $\theta$ is the emission angle with respect to the normal direction of the diffuser surface, (2) the isotropic emission model assuming $d \Omega / d(\cos \theta)=$ constant, and (3) the cosine $\theta$ emission model using $d \Omega / d(\cos \theta)=\cos \theta$. There were no free parameters in the simulation. Fig. 3 compares the simulation results with the experimental data. The measured thickness profile is close to the cosine $\theta$ model (3). The results of simulations give some guidance when scaling the thickness as a function of the distance between gas diffuser and target support foil.

The origin of the asymmetry in the measured thickness profile is not understood. It is unlikely to be an effect of gravity on the target, since the relative non-uniformity remains constant over a wide range of the total thickness. The shape may be partly explained by the fact that the gas is introduced from the bottom of the diffuser system, hence gas molecules may have a larger probability of diffusing out at the bottom rather than the top of the diffuser. 
Table 1: Results of the thickness measurement at different vertical positions. For definition of source positions see Fig. 1.

\begin{tabular}{c|cc}
\hline $\begin{array}{c}\text { Source position from } \\
\text { target center }(\mathrm{mm})\end{array}$ & $\begin{array}{c}\text { Thickness }\left(\mu \mathrm{g} / \mathrm{cm}^{2} \text { per Torr-litre }\right) \\
\mathrm{H}_{2}\end{array}$ & Ne \\
\hline 20 & $3.00 \pm 0.16$ & $31.5 \pm 1.9$ \\
10 & $3.56 \pm 0.18$ & \\
0 & $3.35 \pm 0.17$ & $32.5 \pm 2.0$ \\
-10 & $2.97 \pm 0.15$ & \\
-20 & $2.54 \pm 0.13$ & $24.7 \pm 1.7$ \\
\hline
\end{tabular}

As a method of measuring thicknesses of thin films, the present work can be applied to a relatively wide range of film thicknesses, e.g. a few $\mu \mathrm{g} \cdot \mathrm{cm}^{-2}$ to about $1 \mathrm{mg} \cdot \mathrm{cm}^{-2}$ for protium. The accuracy of the measurement is limited by the uncertainty in the stopping power in most cases, but the relative accuracy can reach a $1 \%$ level. Muon catalyzed fusion could be also used as a monoenergetic alpha (or proton) source, as suggested in $[5,10]$, instead of radioactive isotopes. The present method is applicable to other experiments using thin film targets $[10,11]$.

\section{Acknowledgement}

The authors gratefully acknowledge support of the Natural Sciences and Engineering Research Council of Canada, US National Science Foundation, and the Swiss National Science Foundation. M.C.F. thanks Rotary International, the University of British Columbia, and the Department of Foreign Affairs and International Trade of the Government of Canada for their support.

\section{References}

[1] P. E. Knowles $t$ al., Hyp. Int. 82, 521 (1993).

[2] P. E. Knowles et al., to be published in Nucl. Instr. Meth. A.

[3] See proceedings of this conference and references therein, P.E. Knowles et al., F. Mulhauser et al., G.M. Marshall et al., and R. Jacot-Guillarmod et al.

[4] R. Jacot-Guillarmod et al., proceedings of this conference; V. M. Bystristsky et al., TRIUMF E742 proposal.

[5] M. C. Fujiwara, Master's thesis, University of British Columbia, unpublished (1994).

[6] M. C. Fujiwara $t$ al., proceedings of this conference; M. C. Fujiwara et al., TRIUMF E767 proposal. 
[7] M. C. Fujiwara $e t$ al., to be submitted to Nucl. Instr. Meth.

[8] J. F. Ziegler et al., The Stopping and Range of Ions in Solids (Pergamon Press, New York, 1985)

[9] ICRU Report 49, Stopping Powers and Ranges for Protons and Alpha Particles (International Commission of Radiation Units and Measurements, Bethesda, Maryland, 1993).

[10] P. Strasser $e t$ al., proceedings of this conference.

[11] K. Nagamine, Hyp. Int. 82, 343 (1993). 